\title{
Entropy-Constrained Scalar Quantization and Minimum Entropy with Error Bound by Discrete Wavelet Transforms in Image Compression
}

\author{
Bing-Fei Wu and Hung-Hseng Hsu
}

\begin{abstract}
The global maximum of an entropy function with different decision levels for a three-level scalar quantizer performed after a discrete wavelet transform was derived. Herein, we considered the case of entropy-constrained scalar quantization capable of avoiding many compression ratio reductions as the mean squared error was minimized. We also dealt with the problem of minimum entropy with an error bound, which was referred to as the rate distortion function. For generalized Gaussian distributed input signals, the Shannon bound would decrease monotonically when the parameter of distribution $\gamma$ was to leave from 2 . That is, the Gaussian distributions would contain the highest Shannon bound among the generalized Gaussian distributions. Additionally, we proposed two numerical approaches of the secant and false position methods implemented in real cases to solve the problems of entropy-constrained scalar quantization and minimum entropy with an error bound. The convergence condition of the secant method was also addressed.
\end{abstract}

\section{INTRODUCTION}

$\mathbf{T}$ HE DISCRETE wavelet transform (DWT) is a kind of pyramid subband decomposition that involves the application of a multiresolution representation to image compression to increase image resolution [3], [18]. The advantage of using such a method in image compression could provide high compression ratios (CR's) as well as maintain good image fidelities (see [1] and [23, p. 399]). The value of image compression characterized by the DWT with entropy reduction is further presented in [25]. The Daubechies' filters, which are widely used in data compression, are shown to be compactly supported and orthogonal (see [6] and [7, p. 167]). All the subimages functioning with the DWT, except for the lowest frequency component, are called the detailed images. We derived that the application of scalar quantization (SQ) extended the detailed images since the histograms are modelled to be generalized Gaussian distributed (see [1] and [22, p. 370]).

Although the quantizer provides high capability that is vital to image compression, it is accompanied by some generated distortion, and this can become a trade-off problem. In our previous work [24], we considered the case of the minimum mean

Manuscript received August 25, 1998; revised September 1, 1999 This work was supported by National Science Council under Grant NSC85-2213-E-009-008. The associate editor coordinating the review of this publication and approving it for publication was Dr. Ali N. Akansu.

B.-F. Wu is with the Department of Electrical and Control Engineering, National Chiao Tung University, Hsinchu, Taiwan, R.O.C. (e-mail: bwu@cc.nctu.edu.tw; vhsu@itri.org.tw).

H.-H. Hsu is with the Computer and Communications Research Laboratories, Industrial Technology Research Institute, Hsinchu, Taiwan, R.O.C.

Publisher Item Identifier S 1053-587X(00)02355-2. squared error (MMSE) of the quantizer, which diminishes the error as much as possible by disregarding CR. Since the entropy is considered to be the index of lossless compressibility and the expected value of the information contained in a picture, the optimal choice of quantization error without regard to entropy will lead to a poor compression result, in general. Hence, the concept to use the entropy instead of the mean squared error as the performance index of optimization is followed. However, the minimum entropy occurs when the decision level of the scalar quantizer approaches infinity. This means that the three-level scalar quantizer, which has three output levels, will be reduced to a one-level scalar quantize, and the output of the quantizer is always near zero. Afterward, the quantization error is maximum and equal to the energy of the input signal, in which the quantizer is shown to be turned off and meaningless.

Two ways may be used to remove these obstacles from the idea of constrained optimization [11]; one approach is to introduce MMSE with entropy constrained, and the other is to minimize the entropy with an error bound, which is the same as the concept of rate distortion function in information science [21]. Gish and Pierce [8] showed that MMSE with entropy constrained to the case in which the numbers of decision levels approach infinity, has uniform decision levels regardless of the probability density function (pdf) of the source signals. In particular, if the sources are independent and identically Gaussian distributed, the MMSE with entropy constrained has an entropy that is only 0.255 bits/pixel greater than the Shannon bound. Wood [26] proposed the MMSE with entropy constrained numerically by using a descent algorithm. Berger [4] further described the conditions necessary for the MMSE with entropy constrained. Netravali and Saigal [15] presented another algorithm to solve the problems of MMSE with entropy constrained based on fixed-point considerations. Noll and Zelinski [16] followed Berger's method to deal with the sources with common pdf's. The authors, however, encountered a drawback relating to the convergence conditions constrained in the algorithms [5], which will be overcome in this paper. The novelty of our paper is therefore to introduce both issues of MMSE with entropyconstrained and minimum entropy with an error bound in a three-level scalar quantizer functioning after the DWT from a geometrical perspective.

The object of the MMSE with entropy constrained herein is to calculate the decision levels of a three-level scalar quantizer such that the quantization error is minimum when the entropy is bounded by a specified value $C$. Unlike the case of MMSE, CR is preserved at an acceptable level, and the feasible solution of 
decision levels is a union of two disconnected sets. Two algorithms, called the secant method and the false position method (also called regula falsi) [9], [20] are proposed to obtain the solutions numerically. We also address the convergence condition in the section on the secant method. Through the quantization error bounded by a given value $D$, the concept of minimum entropy to be implemented in real situations is initiated. The feasible solution of decision levels constitutes a connected set. Because of the convexity of the error function not being formulated yet, we suggest applying the false position method to obtain the two boundary points in the set of the decision levels. The quantization errors corresponding to the set of decision levels satisfy the specified bound $D$. Compared with the entropies evaluated at these two boundary points, we pick up the smaller one as the desired minimum entropy. It is also shown that for generalized Gaussian distributed inputs, the Shannon bounds decrease monotonically when the value of $\gamma$, which is referred to as the parameter of distributions, is no longer 2 . In particular, the rate distortion function referred as the minimum entropy function related to the error bounds is higher than the Shannon bound by about 0.06 bits/pixel if the input signal is Laplacian distributed with the variance equal to $(3.2898)^{2}$. Two testbed pictures (Lena and Mandrill) are used to illustrate the theoretical results.

The organization of this paper is as follows. In the next section, the problems of MMSE with entropy constrained and the minimum entropy with an error bound are formulated. The global maximum of the entropy curve in a three-level scalar quantizer performed after the DWT is presented in Section III and provided for further discussions with respect to its property. We notice that the concavity of the entropy function holds for a particular region of decision levels. Therefore, Section IV presents two kinds of numerical approaches called the secant and false position methods to resolve the problem of entropy-constrained SQ. In addition, we consider solving the problem of minimum entropy with an error bound together with the false position method provided to obtain the results numerically presented in Section V. We also compare the rate distortion function with the Shannon bound. In Section VI, two testbed pictures (Lena and Mandrill) are adopted to illustrate the experimental results discussed above. A concise conclusion is made in the last section.

\section{PROBLEM FoRMULATION}

The histograms of the detailed images processed after the DWT can be modeled to be generalized Gaussian-distributed [1]. By using the three-level scalar quantizer applied to these subimages, high compression effects are achieved at the cost of a little distortion. Our previous work [24] indicated that this became the worst value of CR as the MMSE was reached. The first step to resolve the problem is to sacrifice some mean squared error by varying decision levels with the entropy reduced to a specified value, as defined below. Moreover, we are able to maintain the quantization error in a specified value yet reduce the entropy to as minimum as possible.

Consider the three-level scalar quantizer in Fig. 1, where $X$ and $Y$ are the input and output of the quantizer, respectively,
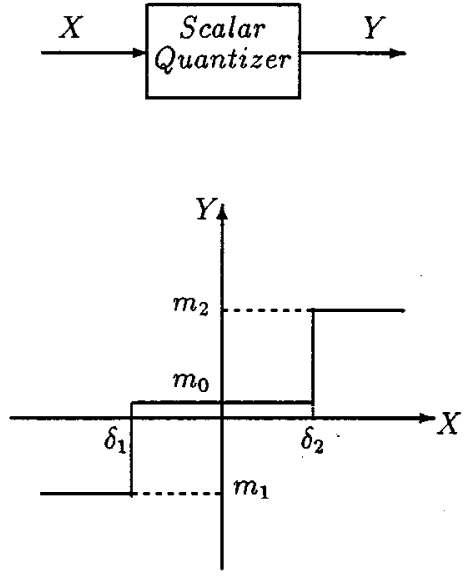

Fig. 1. Input/output characteristic of the three-level scalar quantizer.

$\delta_{1}$ and $\delta_{2}$ are the decision levels, $m_{i}$ for $i=0,1,2$ are the reconstruction levels of the quantizer [2], [10], and $Y \in S=$ : $\left\{m_{j} \mid j=0,1,2\right\}$. The emphasis of this work, which is to minimize the quantization error with entropy constrained and to minimize the entropy with bounded error, will be made by choosing suitable decision and reconstruction levels.

We further consider the special case of $-\delta_{1}=\delta_{2} \triangleq \delta$ to derive the optimal solution provided for reducing one-dimensional complexity in the domain of $\delta$. The mean squared error between the input and output of the special case of the quantizer is defined as

$$
\begin{aligned}
E\left(\delta, m_{1}, m_{0}, m_{2}\right)= & E_{1}\left(\delta, m_{1}\right)+E_{0}\left(\delta, m_{0}\right) \\
& +E_{2}\left(\delta, m_{2}\right)
\end{aligned}
$$

where

$$
\begin{aligned}
& E_{1}\left(\delta, m_{1}\right) \triangleq \int_{-\infty}^{-\delta}\left(x-m_{1}\right)^{2} f_{X}(x) d x \\
& E_{0}\left(\delta, m_{0}\right) \triangleq \int_{-\delta}^{\delta}\left(x-m_{0}\right)^{2} f_{X}(x) d x \\
& E_{2}\left(\delta, m_{2}\right) \triangleq \int_{\delta}^{\infty}\left(x-m_{2}\right)^{2} f_{X}(x) d x
\end{aligned}
$$

and $f_{X}(x)$ is the pdf of the input signal $X$ (see [17, p. 72]). The decision level corresponding to the global minimum error is therefore achieved [24].

Theorem 2.1 [24]: Consider the three-level scalar quantizer with the same decision level. Then, the optimal value of the decision level $\delta^{*}$ to minimize the mean squared error $E$ is

$$
\delta^{*}=\frac{\left(m_{2}^{*}\right)^{2}+\left(m_{1}^{*}\right)^{2}-2\left(m_{0}^{*}\right)^{2}}{2\left(m_{2}^{*}-m_{1}^{*}\right)}
$$

where $m_{j}^{*}$ for $j=0,1,2$ are the centroids of the three levels of the scalar quantizer and are defined as [12], [19]

$$
\begin{aligned}
m_{1}^{*} & =\frac{\int_{-\infty}^{-\delta} x f_{X}(x) d x}{\int_{-\infty}^{-\delta} f_{X}(x) d x} \\
m_{0}^{*} & =\frac{\int_{-\delta}^{\delta} x f_{X}(x) d x}{\int_{-\delta}^{\delta} f_{X}(x) d x} \\
m_{2}^{*} & =\frac{\int_{\delta}^{\infty} x f_{X}(x) d x}{\int_{\delta}^{\infty} f_{X}(x) d x}, \quad \text { respectively. }
\end{aligned}
$$


The histograms of those detailed images of the DWT modelled to be generalized Gaussian distributed are stated as before. The generalized Gaussian law is given explicitly by

$$
f_{X}(x)=a \exp ^{-|b x|^{\gamma}}
$$

with

$$
a=\frac{b \gamma}{2 \Gamma\left(\frac{1}{\gamma}\right)} \quad \text { and } \quad b=\frac{\Gamma\left(\frac{3}{\gamma}\right)^{\frac{1}{2}}}{\sigma \Gamma\left(\frac{1}{\gamma}\right)^{\frac{1}{2}}}
$$

where

$\sigma \quad$ standard deviation of $X$;

$\Gamma(\cdot)$ Gamma function;

$\gamma$ parameter.

The generalized Gaussian law contains two particular cases. i) $\gamma=1$ leads to the Laplacian pdf, and ii) $\gamma=2$ approaches the Gaussian pdf. Obviously, the pdf $f_{X}(x)$ of the input signal $X$ is an even function (symmetric to the origin) with the results of $m_{0}^{*}=0$ and $-m_{1}^{*}=m_{2}^{*} \triangleq m^{*}$ derived. Equation (2) can be reduced to

$$
\delta^{*}=\frac{m^{*}}{2}, \quad \text { where } m^{*}=m_{2}^{*}=\frac{\int_{\delta}^{\infty} x f_{X}(x) d x}{\int_{\delta}^{\infty} f_{X}(x) d x} .
$$

We also proved that $E\left(\delta^{*}\right)$ is the unique global minimum error of the three-level scalar quantizer.

Theorem 2.2 [24]: Consider the three-level scalar quantizer with the same decision level. The mean squared error $E$ defined as (1) has a unique global minimum if $f_{X}(x)$ satisfies the generalized Gaussian law.

Moreover, the entropy of the output of the quantizer is defined as

$$
H_{Y}(\delta)=-\sum_{j=0}^{2} P_{j} \log _{2} P_{j}
$$

where $P_{0}, P_{1}$, and $P_{2}$ are the probabilities of the outputs of the three-level scalar quantizer and are defined as $\int_{-\delta}^{\delta} f_{X}(x) d x$, $\int_{-\infty}^{-\delta} f_{X}(x) d x$, and $\int_{\delta}^{\infty} f_{X}(x) d x$, respectively. These probabilities are functions of the decision level $\delta$ and are independent of the reconstruction levels $m_{j}$ for $j=0,1$ and 2. Equation (5) is equivalent to $-2 P \log _{2} P-(1-2 P) \log _{2}(1-2 P)$, where $P \triangleq P_{2}=P_{1}$. It implies that $P \leq(1 / 2)$. Hence, the criterion of minimization with entropy constrained is

$$
\min _{\delta} E(\delta), \quad \text { subject to } H_{Y}(\delta) \leq C
$$

where $C$ is a specified value.

In addition, we consider the other problem of minimum entropy with an error bound. The criterion is of the form

$$
\min _{\delta} H_{Y}(\delta), \quad \text { subject to } E(\delta) \leq D
$$

where $D$ is a given value.

\section{Global MAXimum OF THE ENTROPY FunCtion}

The entropy, which is the expected value of the information contained in the signal of interest, is an index of compression effect in image coding. We will exactly depict the entropy trajectory of a three-level scalar quantizer prior to numerical approaches provided for solving the constrained optimization problems. The extreme cases $\delta=0$ and $\delta \rightarrow \infty$ of the entropy function are investigated in Lemma 3.1.

Lemma 3.1: Suppose $f_{X}(x)$ is an even function; then, i) $H_{Y}(0)=1$, and ii) $\lim _{\delta \rightarrow \infty} H_{Y}(\delta) \rightarrow 0$. More precisely, the three-level scalar quantizer in cases i) and ii) are reduced to twoand one-level scalar quantizers, respectively.

Proof: i) When $\delta=0$, the term $(1-2 P) \log _{2}(1-2 P)$ will disappear because of the vanishment of $(1-2 P)$. The value of $-2 P \log _{2} P$ is equal to 1 since $P=(1 / 2)$. Hence, $H_{Y}(0)=1$. ii) The value of $P$ approaches zero as $\delta \rightarrow \infty$, implying that $-2 P \log _{2} P \rightarrow 0$. Moreover, the term $-(1-2 P) \log _{2}(1-2 P)$ approaches zero as $(1-2 P) \rightarrow 1$.

Lemma 3.2: Suppose $f_{X}(x)$ is an even function; then, $P(\delta)$ is a decreasing function of $\delta$.

Proof: Taking the derivative of $P$ with respect to $\delta$

$$
\frac{d P(\delta)}{d \delta}=\frac{d \int_{\delta}^{\infty} f_{X}(x) d x}{d \delta}=-f_{X}(\delta)<0 .
$$

Next, taking the derivative of the entropy function with respect to $\delta$, we obtain

$$
\begin{aligned}
\frac{d H_{Y}}{d \delta}= & 2 f_{X}(\delta) \log _{2} \int_{\delta}^{\infty} f_{X}(x) d x \\
& +2 \int_{\delta}^{\infty} f_{X}(x) d x \frac{f_{X}(\delta)}{\ln 2 \int_{\delta}^{\infty} f_{X}(x) d x} \\
& -2 f_{X}(\delta) \log _{2}\left(1-2 \int_{\delta}^{\infty} f_{X}(x) d x\right) \\
& -\left(1-2 \int_{\delta}^{\infty} f_{X}(x) d x\right) \frac{2 f_{X}(\delta)}{\ln 2\left(1-2 \int_{\delta}^{\infty} f_{X}(x) d x\right)} \\
= & 2 f_{X}(\delta) \log _{2} \frac{\int_{\delta}^{\infty} f_{X}(x) d x}{\left(1-2 \int_{\delta}^{\infty} f_{X}(x) d x\right)} \\
= & 2 f_{X}(\delta) \log _{2} \frac{P}{1-2 P} .
\end{aligned}
$$

In Lemma 3.3, we are going to illustrate the characteristics of the derivative of entropy when $\delta=0$ and $\delta \rightarrow \infty$.

Lemma 3.3: Suppose $f_{X}(x)$ is generalized Gaussian distributed; then, i) $\left.\left(d H_{Y}(\delta) / d \delta\right)\right|_{\delta=0}=\infty$, and ii) $\lim _{\delta \rightarrow \infty}\left(d H_{Y}(\delta) / d \delta\right) \rightarrow 0$.

Proof: i) If $\delta=0$, then $P=(1 / 2)$. It implies that $\left.\left(d H_{Y}(\delta) / d \delta\right)\right|_{\delta=0}=\infty$ since $\left(d H_{Y} / d \delta\right)=$ $2 f_{X}(\delta) \log _{2}(P / 1-2 P)$ in (6). ii) Based on the non-negativeness of $\log _{2}(1 /(1-2 P))$, the following inequalities are obtained:

$$
f_{X}(\delta) \log _{2} P \leq f_{X}(\delta) \log _{2} \frac{P}{1-2 P}<0, \quad \text { for } P \leq \frac{1}{3} .
$$

In addition, the limiting value of $f_{X}(\delta) \log _{2} P$ as $\delta \rightarrow \infty$ is checked as

$$
\begin{aligned}
\lim _{\delta \rightarrow \infty} f_{X}(\delta) \log _{2} P \\
=\lim _{\delta \rightarrow \infty} \frac{f_{X}(\delta) \ln P}{\ln 2} \\
=\lim _{\delta \rightarrow \infty} \frac{\frac{-f_{X}(\delta)}{P}}{\ln 2 \frac{-f_{X}^{\prime}(\delta)}{f_{X}^{2}(\delta)}}, \quad \text { where } f_{X}^{\prime} \text { is the derivative of } f_{X} \\
=\frac{1}{2} \lim _{\delta \rightarrow \infty} \frac{2 f_{X}(\delta) f_{X}^{\prime}(\delta)}{-\gamma(\gamma-1) b^{2}(b \delta)^{\gamma-2} P+\gamma b(b \delta)^{\gamma-1} f_{X}(\delta)}
\end{aligned}
$$




$$
\begin{aligned}
& =\frac{1}{2} \lim _{\delta \rightarrow \infty} \frac{-2 f_{X}^{2}(\delta)-4 \delta f_{X}(\delta) f_{X}^{\prime}(\delta)}{(\gamma-1) f_{X}(\delta)+f_{X}(\delta)+\delta f_{X}^{\prime}(\delta)} \\
& =\frac{1}{2} \lim _{\delta \rightarrow \infty} \frac{-2 f_{X}(\delta)\left(1-2 \gamma(b \delta)^{\gamma}\right)}{\gamma\left(1-(b \delta)^{\gamma}\right)} \\
& \rightarrow 0 .
\end{aligned}
$$

Because the decay of the exponential term $f_{X}(\delta)$ is faster than that of the polynomial term $(b \delta)^{\gamma}$, the last equality approaches zero. By (7), the result of ii) is obtained.

In addition, let the value of the decision level be defined as $\delta_{H}^{*}$ such that $P\left(\delta_{H}^{*}\right)=(1 / 3)$. Explicitly, by Lemma 3.2, the derivative of the entropy in (6) is greater than zero for $\delta<\delta_{H}^{*}$, equal to zero for $\delta=\delta_{H}^{*}$, and negative otherwise. As a result, Lemma 3.4 is apparently obtained.

Lemma 3.4: Suppose that $f_{X}(x)$ is generalized Gaussian distributed. The derivative of entropy $\left(d H_{Y} / d \delta\right)$ is greater than zero for $\delta<\delta_{H}^{*}$, equal to zero for $\delta=\delta_{H}^{*}$, and less than zero for $\delta>\delta_{H}^{*}$

By the lemmas mentioned above, the global maximum of the entropy function will be shown in the following theorem.

Theorem 3.1: Suppose $f_{X}(x)$ is generalized Gaussian distributed. The entropy of the output signals in Fig. 1 has a global maximum $H_{Y}^{*}=\log _{2} 3$.

Proof of Theorem 3.1: To demonstrate the property of the global maximum of the entropy function, the curve of entropy is traced from $\delta=0$. By Lemma 3.1, we have $H_{Y}(0)=1$. When $\delta$ departs from $0, H_{Y}(\delta)$ increases from 1 based on the positive value of $\left(d H_{Y} / d \delta\right)$ derived in Lemmas 3.3 and 3.4. The increment of $H_{Y}(\delta)$ will be stopped if the optimal value of the decision value occurs, i.e., $\delta=\delta_{H}^{*}$. Sequentially, $H_{Y}(\delta)$ becomes a strictly decreasing function for $\delta>\delta_{H}^{*}$ if the value of $\left(d H_{Y} / d \delta\right)$ in Lemma 3.4 is negative. While the entropy function decays slowly and approaches zero as $\delta \rightarrow \infty$, we can precisely observe the global maximum property of entropy in Fig. 2. Hence, the entropy function has a global maximum. The value of maximum entropy $H_{Y}^{*}$ is $-3 \times(1 / 3) \log _{2}(1 / 3)=$ $\log _{2} 3 \approx 1.5850$.

\section{Minimum QuANTIZATION ERROR WITH ENTROPY CONSTRAINED}

We have shown the global minimum of quantization error and the global maximum of entropy in scalar quantization. In this section, the complete procedures will be presented with the observation of the geometrical point to obtain the minimum quantization error with entropy constrained. We suppose that the value of entropy bound $C$ is less than the maximum entropy $\log _{2} 3$. There are two intersection points generated in the entropy function when the horizontal line, which represents the value of entropy equal to $C$, is introduced. The values of $\delta$ at these two points are denoted as $\delta_{H}^{C_{1}}$ and $\delta_{H}^{C_{2}}$, respectively, and lie in the two sides of $\delta_{H}^{*}$. All values of $\delta$ satisfied to the entropy constraint $H_{Y} \leq C$ are subject to the set $S_{C}=\left\{\delta \mid \delta \leq \delta_{H}^{C_{1}}\right.$ or $\left.\delta \geq \delta_{H}^{C_{2}}\right\}$. Furthermore, the assumption made under $\delta_{H}^{C_{1}}<$ $\delta_{H}^{*}<\delta_{H}^{C_{2}}$ is reasonable. Specially, if $C<1$, there exists only one intersection point $\delta_{H}^{C_{2}}$. Hence, $S_{C}$ will be reduced to $S_{C_{2}}=\left\{\delta \mid \delta \geq \delta_{H}^{C_{2}}\right\}$ and $S_{C_{1}}$ defined as $\left\{\delta \mid \delta \leq \delta_{H}^{C_{1}}\right\}$. We will investigate $\delta_{H}^{C_{1}}$ and $\delta_{H}^{C_{2}}$ by using the secant method and the false position method, respectively.

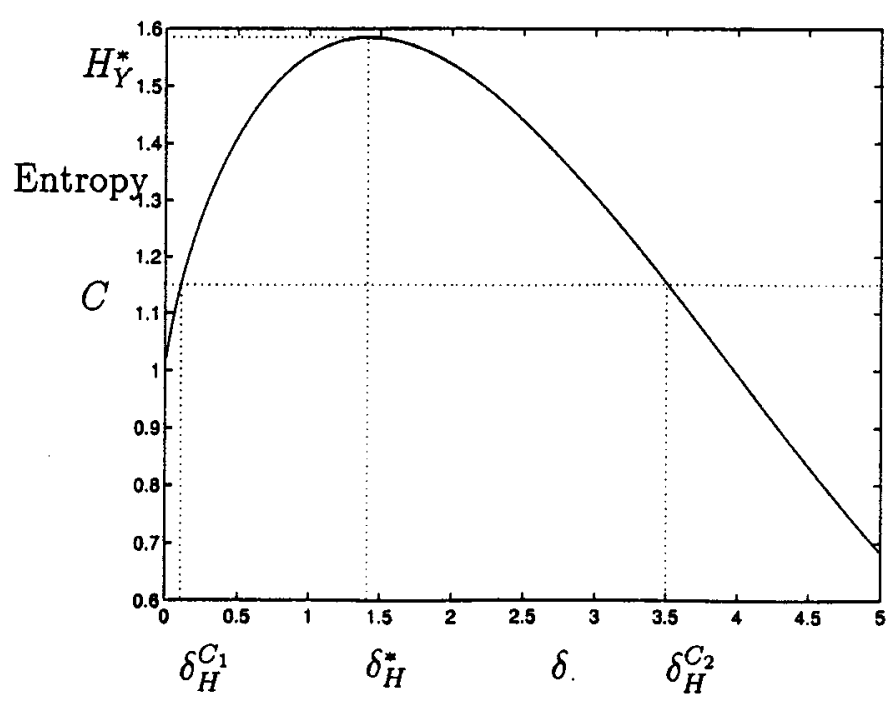

Fig. 2. Global maximum of $H_{Y}$.

\section{A. Solving $\delta_{H}^{C_{1}}$ by the Secant Method}

In this subsection, we propose an algorithm to achieve the value of $\delta_{H}^{C_{1}}$ by the secant method. The convergence condition of this algorithm is also discussed. Although the secant method will converge almost quadratically (see [12, p. 68]), it may be diverged if the curve of entropy has inflection points. To preserve the convergence global, the curve needs to be either concave or convex.

Theorem 4.1: Supposing that $f_{X}(x)$ is generalized Gaussian distributed, the entropy of the output signals in Fig. 1 is shown to be concave for $\delta \leq \delta_{H}^{*}$.

Proof of Theorem 4.1: Taking the second derivative of $H_{Y}(\delta)$

$$
\begin{aligned}
\frac{d^{2} H_{Y}(\delta)}{d \delta^{2}} & \\
= & -2 \gamma b(b \delta)^{\gamma-1} f_{X}(\delta) \log _{2} \frac{P}{1-2 P} \\
& +2 f_{X}(\delta) \frac{1-2 P}{P} \times \frac{-f_{X}(\delta)(1-2 P)-2 f_{X}(\delta) P}{(\ln 2)(1-2 P)^{2}} \\
= & f_{X}(\delta)\left\{-2 \gamma b(b \delta)^{\gamma-1} \log _{2} \frac{P}{1-2 P}\right. \\
& \left.-2 f_{X}(\delta) \frac{1}{(\ln 2) P(1-2 P)}\right\} .
\end{aligned}
$$

If $\delta \leq \delta_{H}^{*}$, i.e., $P \geq(1 / 3)$, then $\left(d^{2} H_{Y}(\delta) / d \delta^{2}\right)<0$. Hence, the concavity of $H_{Y}(\delta)$ for $\delta \leq \delta_{H}^{*}$ is sustained. The proof is completed.

Remark: By Lemmas 3.3, 3.4, and Theorem 4.1, the derivative of the entropy $\left(d H_{Y} / d \delta\right)$ (see Fig. 3) strictly decreases to zero from $\infty$ for $\delta \leq \delta_{H}^{*}$. When $\delta>\delta_{H}^{*}$, the sign of $\left(d^{2} H_{Y}(\delta) / d \delta^{2}\right)$ is not definite, and this implies that there exists at least one inflection point in the entropy function.

By the concavity of $H_{Y}(\delta)$, we can develop the following algorithm to reach the value of $\delta_{H}^{C_{1}}$.

$$
\begin{aligned}
\delta_{H}^{1} & =\delta_{H}^{0}+\frac{1}{\beta}\left[C-H_{Y}\left(\delta_{H}^{0}\right)\right] \\
\delta_{H}^{k+1} & =\delta_{H}^{k}+\frac{\delta_{H}^{k}-\delta_{H}^{k-1}}{H_{Y}\left(\delta_{H}^{k}\right)-H_{Y}\left(\delta_{H}^{k-1}\right)}\left[C-H_{Y}\left(\delta_{H}^{k}\right)\right]
\end{aligned}
$$




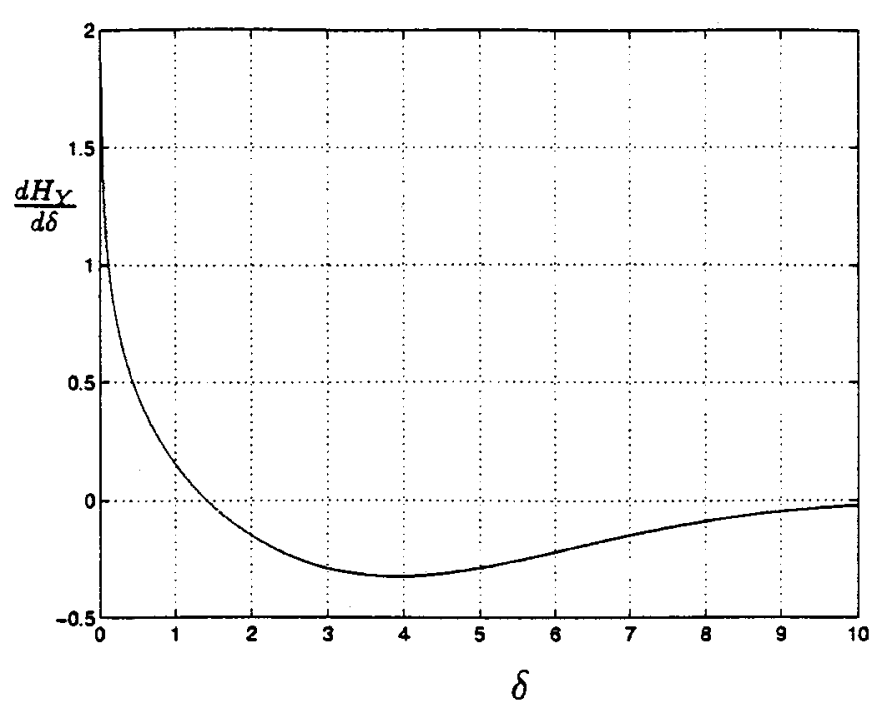

Fig. 3. Derivative of the entropy function.

where $\delta_{H}^{0}$, which is less than $\delta_{H}^{C_{1}}$, is the first initial condition of the secant method and is always set to be a small positive number for stability. The second initial condition $\delta_{H}^{1}$ is constituted by (8). The value of $\beta$ is selected to be $\left(H_{Y}\left(\delta_{H}^{0}\right)-1 / \delta_{H}^{0}\right)$ to ensure that $\delta_{H}^{1}$ lies in the interval of $\left[\delta_{H}^{0}, \delta_{H}^{C_{1}}\right]$. See the following discussions for more details.

\section{Convergence Condition of the Secant Method}

1) Define $\tilde{\delta}_{H}^{k}=\delta_{H}^{C_{1}}-\delta_{H}^{k}$. Then

$$
\frac{\tilde{\delta}_{H}^{1}}{\tilde{\delta}_{H}^{0}}=\frac{\delta_{H}^{C_{1}}-\delta_{H}^{1}}{\delta_{H}^{C_{1}}-\delta_{H}^{0}}=1-\frac{C-H_{Y}\left(\delta_{H}^{0}\right)}{\beta\left(\delta_{H}^{C_{1}}-\delta_{H}^{0}\right)} .
$$

Since $H_{Y}(\delta)$ is a concave function, for $\delta<\delta_{H}^{C_{1}} \leq \delta_{H}^{*}$

$$
\begin{aligned}
0 & <\frac{C-H_{Y}\left(\delta_{H}^{0}\right)}{\delta_{H}^{C_{1}}-\delta_{H}^{0}}<\frac{d H_{Y}\left(\delta_{H}^{0}\right)}{d \delta}<\frac{H_{Y}\left(\delta_{H}^{0}\right)-1}{\delta_{H}^{0}} \\
& =\beta, \quad \text { for } \delta_{H}^{0}<\delta_{H}^{C_{1}} .
\end{aligned}
$$

Hence, we obtain $0<\left(\tilde{\delta}_{H}^{1} / \tilde{\delta}_{H}^{0}\right)<1$, which implies that $\delta_{H}^{1}$ lies in the interval of $\left[\delta_{H}^{0}, \delta_{H}^{C_{1}}\right]$.

2) The convergence condition for $k \geq 1$ can be addressed as

$$
\begin{aligned}
\frac{\tilde{\delta}_{H}^{k+1}}{\tilde{\delta}_{H}^{k}} & =\frac{\delta_{H}^{C_{1}}-\delta_{H}^{k+1}}{\delta_{H}^{C_{1}}-\delta_{H}^{k}} \\
& =1-\frac{\frac{C-H_{Y}\left(\delta_{H}^{k}\right)}{\left(\delta_{H}^{C_{1}}-\delta_{H}^{k}\right)}}{\frac{H_{Y}\left(\delta_{H}^{k}\right)-H_{Y}\left(\delta_{H}^{k-1}\right)}{\left(\delta_{H}^{k}-\delta_{H}^{k-1}\right)}} \\
& <1 .
\end{aligned}
$$

Hence, we obtain $0<\left(\tilde{\delta}_{H}^{k+1} / \tilde{\delta}_{H}^{k}\right)<1$, which implies that $\delta_{H}^{k+1}$ lies in the interval of $\left[\delta_{H}^{k}, \delta_{H}^{C_{1}}\right]$.

Based on the result, the value in the algorithm from (9) will certainly converge to $\delta_{H}^{C_{1}}$.

\section{B. Solving $\delta_{H}^{C_{2}}$ by the False Position Method}

We introduce the false position method instead of the secant method provided to obtain $\delta_{H}^{C_{2}}$ since there exists at least one inflection point for $\delta>\delta_{H}^{*}$. With the false position method applied on the entropy function, the convergence stability is ro- (a)

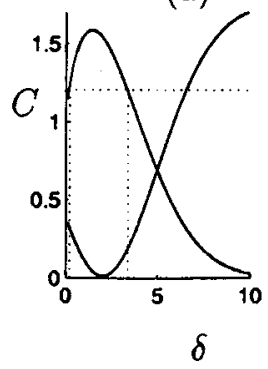

(b)

(c)
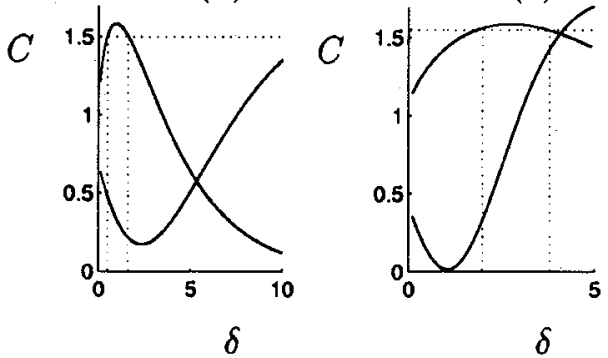

Fig. 4. Three cases of minimum error with entropy constrained. (a) $\delta_{H}^{C 1}<$ $\delta^{*}<\delta_{H}^{C_{2}}$. (b) $\delta_{H}^{C_{1}}<\delta_{H}^{C_{2}}<\delta^{*}$. (c) $\delta^{*}<\delta_{H}^{C_{1}}<\delta_{H}^{C_{2}}$.

bustly preserved, although the convergence rate is linear only. The procedure to find $\delta_{H}^{C_{2}}$ is developed as follows.

1) Find $\delta_{a}$ such that $\delta_{a}>\delta_{H}^{C_{1}}$ and $H_{Y}\left(\delta_{a}\right)>C$. If $C<1$, $\delta_{H}^{C_{1}}$ will vanish with $\delta_{a}$ set to be zero for convenience.

2) $\delta_{b}$ is formed with a positive value added to $\delta_{a}$ under the condition of $H_{Y}\left(\delta_{b}\right)<C$.

3)

$$
\delta_{n}=\frac{\delta_{b}\left[H_{Y}\left(\delta_{a}\right)-C\right]-\delta_{a}\left[H_{Y}\left(\delta_{b}\right)-C\right]}{H_{Y}\left(\delta_{a}\right)-H_{Y}\left(\delta_{b}\right)} .
$$

4) $\delta_{a}$ will be replaced by $\delta_{n}$ if the sign of $H_{Y}\left(\delta_{a}\right)-C$ is identical to that of $H_{Y}\left(\delta_{n}\right)-C$; otherwise, $\delta_{b}$ is substituted by $\delta_{n}$.

5) Continue the procedure until the value of $\left|H_{Y}\left(\delta_{n}\right)-C\right|$ is smaller than the specified small value and $H_{Y}\left(\delta_{n}\right) \leq C$.

\section{Finding the Minimum Quantization Error from $S_{C}$}

Since $S_{C}$ is clearly identified when $\delta_{H}^{C_{1}}$ and $\delta_{H}^{C_{2}}$ are located, we are going to determine the minimum quantization error found in $S_{C}$. There are three conditions to be considered as follows, and these are shown in Fig. 4.

1) $\delta_{H}^{C_{1}}<\delta^{*}<\delta_{H}^{C_{2}}$ :

Comparing the quantization error of $\delta_{H}^{C_{1}}$ with that of $\delta_{H}^{C_{2}}$, the smaller one is of interest.

2) $\delta_{H}^{C_{1}}<\delta_{H}^{C_{2}}<\delta^{*}$ :

$\delta^{*}$ is selected since $\delta^{*} \in S_{C_{2}} \subset S_{C}$.

3) $\delta^{*}<\delta_{H}^{C_{1}}<\delta_{H}^{C_{2}}$ :

$\delta^{*}$ is selected since $\delta^{*} \in S_{C_{1}} \subset S_{C}$.

Remark: For the particular case of $C<1$, the problem is reduced to the cases of $\delta_{H}^{C_{2}}<\delta^{*}$ and $\delta_{H}^{C_{2}}>\delta^{*}$. As a result, $\delta_{H}^{C_{2}}$ is chosen if $\delta^{*}$ does not belong to the set $S_{C_{2}} \equiv S_{C}$; otherwise, $\delta^{*}$ is preferred.

\section{Minimum ENTROPY WITH AN ERROR BOUND}

The problem of minimum entropy with an error bound in a three-level scalar quantizer will be explored similarly from the geometrical perspective. This means that the entropy is obtained as minimum as possible under the condition that the quantization error does not exceed the specified value. We suppose that the bounded value of the quantization error is set at $D$ and that there is absolutely no solution if $D<E\left(\delta^{*}\right)$, where $E\left(\delta^{*}\right)$ is the minimum quantization error. In general, only two points are generated in the error curve when the error is exactly approximate to $D$. The values of the decision levels are defined 


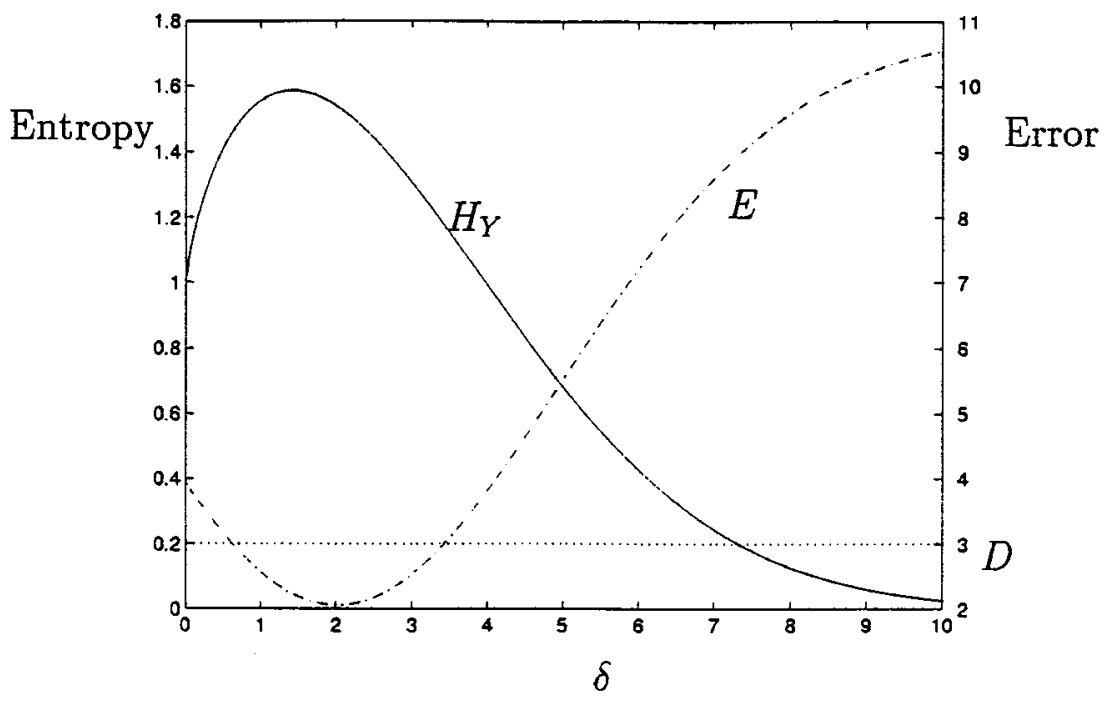

Fig. 5. Minimum entropy with an error bound.

to be $\delta_{E}^{D_{1}}$ and $\delta_{E}^{D_{2}}$, where $\delta_{E}^{D_{1}}<\delta_{E}^{D_{2}}$, which implies that $\delta_{E}^{D_{1}}<\delta^{*}<\delta_{E}^{D_{2}}$. In the same manner, $S_{D}$ is defined to be $\left\{\delta \mid \delta_{E}^{D_{1}} \leq \delta \leq \delta_{E}^{D_{2}}\right\}$ and is a connected set. Unlike the concavity of the entropy function for $\delta<\delta_{H}^{*}$, the convexity of the error function is not sustained. Hence, the false position method is adopted to achieve both values of $\delta_{E_{1}}^{D_{1}}$ and $\delta_{E}^{D_{2}}$ independently. After obtaining the two values of $\delta_{E}^{D_{i}}$ for $i=1,2$, there is only one condition remaining to be considered, and this is more simple than that of the minimum problem with entropy constrained restricted to three conditions. The value of interest to this research is the one whose entropy $H_{Y}\left(\delta_{E}^{D_{i}}\right)$ is the smallest. See Fig. 5 for more details.

Remark: The fact that the error of the energy is equal to the difference between the input energy and output energy is demonstrated in [24]. It is formulated as

$$
E(\delta)=R_{X}(0)-R_{\tilde{Y}}(0)
$$

where $R_{X}(0) \triangleq \int_{-\infty}^{\infty} x^{2} f_{X}(x) d x$ is the energy of the input signal, $X, R_{\tilde{Y}}(0) \triangleq\left(m_{1}^{*}\right)^{2} \int_{-\infty}^{\delta_{1}} f_{X}(x) d x+$ $\left(m_{0}^{*}\right)^{2} \int_{\delta_{1}}^{\delta_{2}} f_{X}(x) d x+\left(m_{2}^{*}\right)^{2} \int_{\delta_{2}}^{\infty} f_{X}(x) d x$, and $\tilde{Y}$ is defined as the output of the three-level scalar quantizer while the optimal reconstruction levels are selected. The error function evaluated at $\delta=0, E(0)$ is equal to $R_{X}(0)-(1 / 2)\left(m_{1}^{*}\right)^{2}-(1 / 2)\left(m_{2}^{*}\right)^{2}$ with value smaller than that of $E(\infty)=R_{X}(0)$. Hence, there exists only one intersection point in the problem of minimum entropy with an error bound if $D$ is larger than the value of $E(0)$. The set $S_{D}$ is represented as $\left\{\delta \mid 0 \leq \delta \leq \delta_{E}^{D_{2}}\right\}$, which reveals that the minimum entropy herein will be obtained by comparing the value of $H_{Y}\left(\delta_{E}^{D_{2}}\right)$ with 1 , which is the value of $H_{Y}(0)$. The smaller one is of interest.

\section{A. The Rate Distortion Function}

For a given error bound, the minimum entropy is obtained with the three-level SQ performed on the DWT. Based on the result, we attempt to plot the curve of entropy with respect to the error bounds, which is also called the rate distortion function.
Suppose the input signal $X$ is generalized Gaussian distributed; then, from (3), the pdf of $X$ is

$$
f_{X}(x)=a \exp ^{-|b x|^{\gamma}} \text {. }
$$

Hence, the entropy of $X$ is

$$
\begin{aligned}
H_{X} & =-\int_{-\infty}^{\infty} f_{X}(x) \log _{2} f_{X}(x) d x \\
& =\log _{2} \frac{e^{\frac{1}{\gamma}}}{a}
\end{aligned}
$$

Shannon's bound of the rate distortion function is defined as [21]

$$
R(E)=\frac{1}{2} \log _{2} \frac{V_{0}}{E}
$$

where $V_{0}$ is the entropy variance corresponding to $H_{X}$, that is, the variance of a Gaussian distribution having the same entropy $H_{X}$. We therefore have

$$
H_{X}=\frac{1}{2} \log _{2}\left(2 \pi e V_{0}\right) .
$$

From (10) and (12), we get

$$
V_{0}=\frac{e^{\frac{2}{\gamma}}}{2 \pi e a^{2}}=\frac{2 \sigma^{2} \Gamma^{3}\left(\frac{1}{\gamma}\right) e^{\frac{2}{\gamma}}}{\pi e \gamma^{2} \Gamma\left(\frac{3}{\gamma}\right)} .
$$

Equation (13) shows that the entropy variance is a function of $\gamma$. The discussion about the trajectory of the entropy variance is observed in the following theorem.

Theorem 5.1: Suppose the signal $X$ is generalized Gaussian distributed, then, the entropy variance $V_{0}$ corresponding to the input signal $X$ has a global maximum when $\gamma=2$, where $\gamma$ is the parameter of the generalized Gaussian distributions and is greater than zero. It also shows that the entropy variance increases monotonically from 0 to $\sigma^{2}$ for $\gamma \leq 2$ and decreases monotonically for $\gamma>2$.

Proof of Theorem 5.1: See the Appendix for more details. The function of entropy variance is shown in Fig. 6 with the values of $\gamma$ varying from 0.001 to 5 .

Remark: By definition of the Shannon bound in (11), we notice that the Shannon bound is proportional to the entropy vari- 


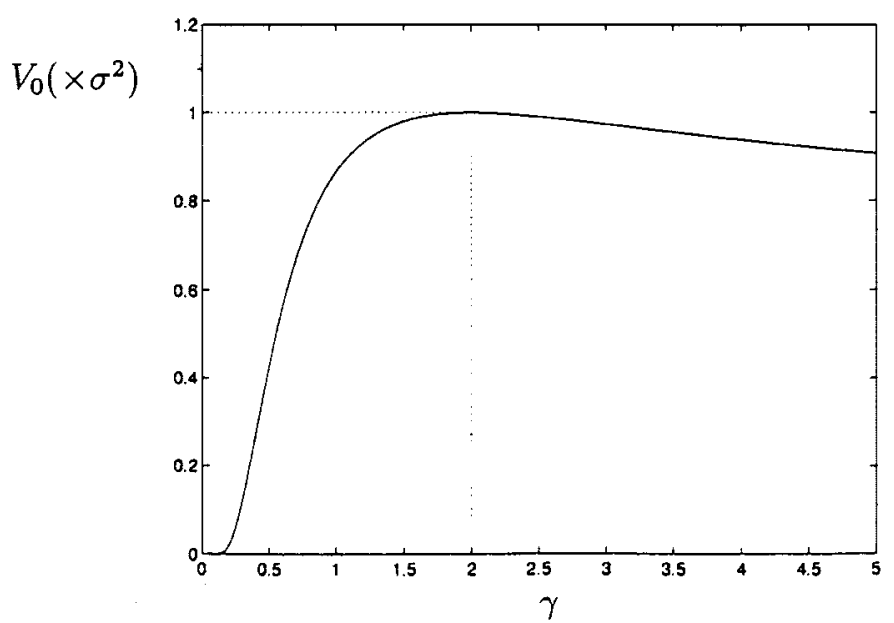

Fig. 6. Maximum of the entropy variance occurs when $\gamma=2$.

ance. Hence, it is concluded that the Shannon bound decreases monotonically when the values of $\gamma$ is no longer 2 .

In particular, we consider the case of Laplacian distribution with pdf of the form

$$
f_{X}(x)=\frac{1}{\sqrt{2} \sigma} \exp ^{-\frac{\sqrt{2}}{\sigma}|x|} .
$$

Therefore, the entropy of $X$ is

$$
H_{X}=\frac{1}{2} \log _{2} 2 \sigma^{2} e^{2} .
$$

To compare the rate distortion function of Laplacian distributions with that of $D_{h 1}$ in Lena, we select the variance to be $(3.2898)^{2}$, which is identical to the variance of $D_{h 1}$. The comparison of the entropy function with the Shannon bound is shown in Fig. 7. It points out that the rate distortion function is greater than the Shannon bound by about 0.06 bits/pixel for an entropy of below 0.4 bits/pixel.

\section{EXPERIMENTAL RESULTS}

Examples of two two-dimensional images (Lena and Mandrill) are presented to illustrate the theoretical results mentioned before. The size of the testbed images is $512 \times 512$ pixels with 8-bit gray levels. The Daubechies' filter with length 20 is adopted in the DWT decomposition since it is orthogonal and compactly supported (see [6] and [7, p. 167]). This structure follows Mallat's algorithm [13], which deals with the two-dimensional image problem and belongs to some kind of pyramid subband codings. $A_{1}$ represents the lowest frequency subimage of the first layer [resolution (1/2)] DWT decomposition. $D_{h 1}, D_{v 1}$ and $D_{d 1}$ are the horizontal, vertical, and diagonal oriented subimages with resolution $(1 / 2)$, respectively, which are referred to as detailed images. Given such a structure, we are then able to derive the three-layer DWT decomposition.

\section{A. Minimum Error with Entropy Constrained}

The detailed images $D_{h 1}$ in Lena and Mandrill are illustrated to determine the minimum quantization error with the entropy

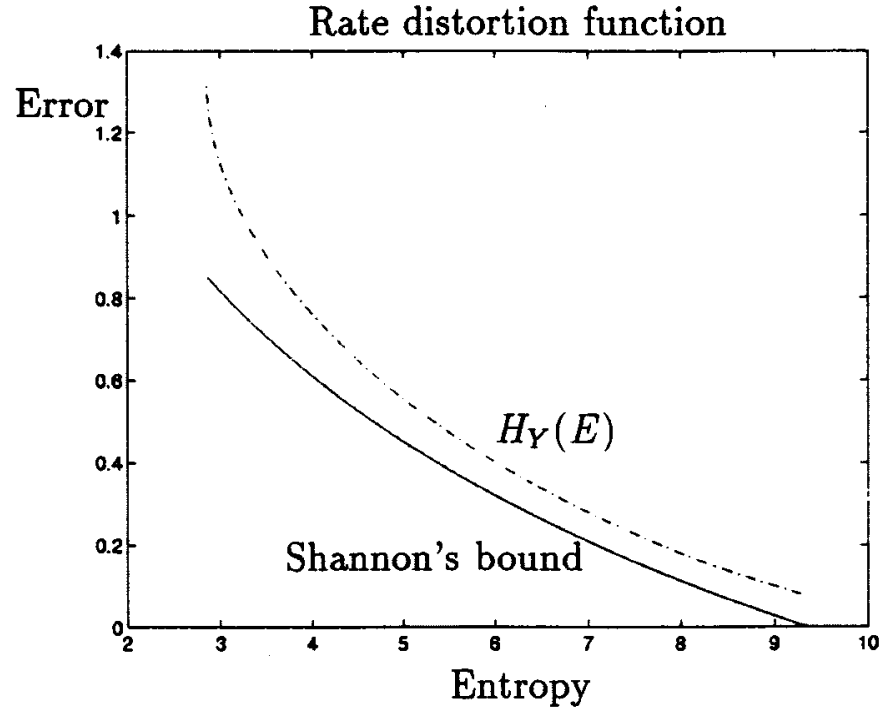

Fig. 7. Minimum entropy function with different error bounds is greater than the Shannon bound by about 0.06 bits/pixel if the source is Laplacian distributed with $\sigma=3.2898$.

constrained. The functions of entropy and quantization error are depicted in Figs. 8 and 9, where the properties of the global maximum and minimum of the entropy and quantization error are shown, respectively.

By the concavity of the entropy function for $\delta<\delta_{H}^{*}$, we use the available secant method to find the value of $\delta_{H}^{C_{1}}$. In Table I, the value at 0.3415 is found to determine the set $S_{C_{1}}$ for the testbed picture of Lena. Next, we check whether the decision value corresponding to the global minimum error $\delta^{*}$ is contained in the set $S_{C_{1}}$ or not. By adding a tiny value to 0.3415 (e.g., 0.0001), the value of the quantization error is shown to have decreased. We know that $\delta^{*}$ is not in $S_{C_{1}}$, and the value of the minimum error in this set is 6.7011 when the entropy bound $C=1.5$ is reached.

The results of another case for $\delta>\delta_{H}^{*}$ is derived in Table II by using the false position method. The value of $\delta_{H}^{C_{2}}$ is found to be 1.0658 to determine the set $S_{C_{2}}$. The set $S_{C}$ is the union of two separable sets $S_{C_{1}}$ and $S_{C_{2}}$. Similarly, the location of $\delta^{*}$ has had to be fixed such that the smaller value of the quantization error is generated if $\delta$ is substituted by a larger value 1.0657. This implies that $\delta^{*}=3.912$ does lie in the set $S_{C_{2}}$, and the minimum error in this set is 4.2120, which is consistent with Condition 2 in Section IV-C. As a result, the minimum quantization error with entropy constrained is 4.2120 , which is simply the minimum quantization error with no constraint [24]. The experimental results, which are functioned in the testbed picture of Mandrill, are also listed in Tables I and II, and they are consistent with Condition 2 after taking the same test performed as above. The minimum quantization error with entropy bounded below or equal to 1.5 is 15.2646 with $\delta^{*}=5.611$. In the case of changing the entropy constraint to 1.05 , the optimal decision value in set $S_{C}$ is no longer $\delta^{*}$. It should have been $\delta_{H}^{C_{2}}$ with $C=1.05$, as illustrated in Fig. 9. The comparison reveals that the image compression condensed in the case of Mandrill is harder than that of Lena. 


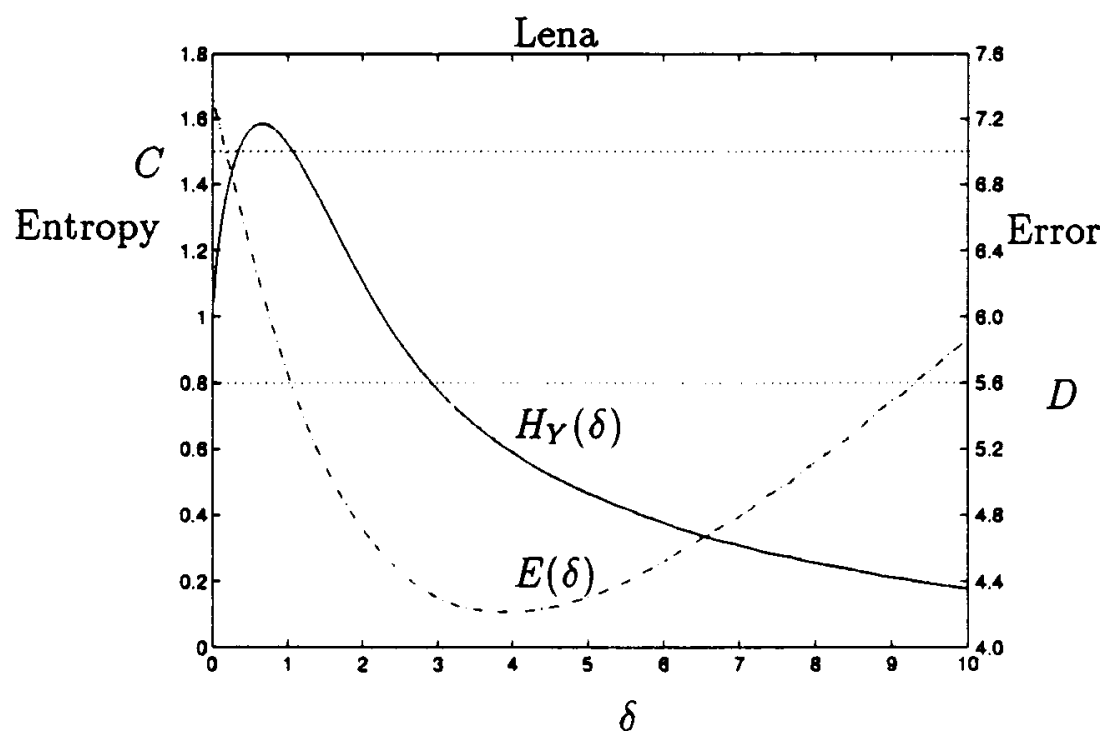

Fig. 8. Entropy and quantization error functions of $D_{h_{1}}$ in Lena after the DWT.

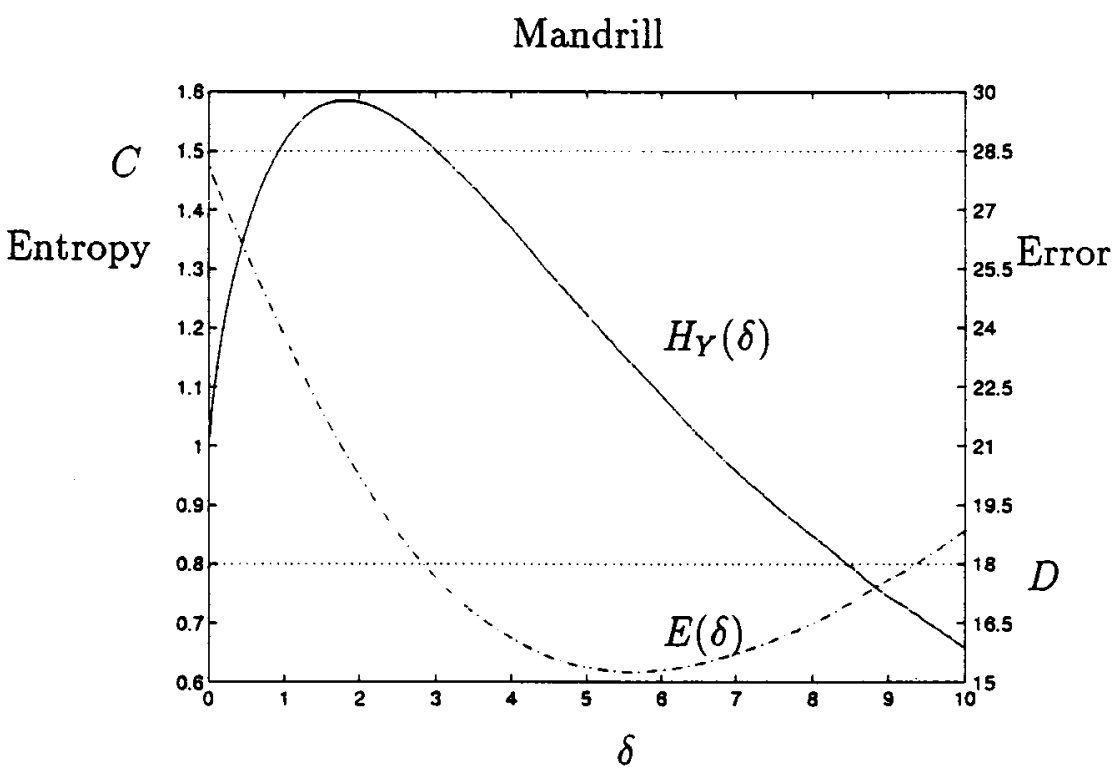

Fig. 9. Entropy and quantization error functions of $D_{h_{1} 1}$ in Mandrill after the DWT.

\section{B. Minimum Entropy with an Error Bound}

The false position method is provided to solve the values of $\delta_{E}^{D_{1}}$ and $\delta_{E}^{D_{2}}$ since the convexity of the error function does not hold, in general. Comparatively, the convergence condition of this numerical approach is simple [9]. The initializations of $\delta_{a}$ and $\delta_{b}$ are located properly such that the corresponding quantization error must be located on the opposite sides of $D$. For the cases of $D_{h 1}$ in Lena and Mandrill, the experimental results with tolerance equal to 0.001 are shown in Tables III and IV. The error bounds are set to be 5.6 and 18 in Lena and Mandrill, respectively. In the case of Lena, the entropy of interest is 0.2009 , which is smaller than 1.5093. On the other hand, the entropy is 0.7145, which is smaller than 1.5196 in Mandrill.

Moreover, the rate distortion function of $D_{h 1}$ in Lena processed with the three-level scalar quantizer is addressed as follows. By experimental results, the values of entropy and vari- ance are about 3.2804 and $(3.2898)^{2}$, respectively. Next, we calculate the entropy variance $V_{0}$ by

$$
H_{D_{h 1}}=3.2804=\frac{1}{2} \log _{2}\left(2 \pi e V_{0}\right) .
$$

As a result, $V_{0} \approx 5.5274$. The Shannon bound $R(E)$ obtained from (11) is compared with the entropy function $H_{Y}(E)$, as shown in Fig. 10. It reveals that the three-level scalar quantizer performs well after the DWT since the rate distortion function is only 0.2 bits/pixel greater than the Shannon bound. From Figs. 7 and 10, we observe that the rate distortion function $H_{Y}(E)$ and the Shannon bound with Laplacian-distributed inputs are higher than those of the case in $D_{h 1}$. The observations are reasonable since the histogram of $D_{h 1}$ in Lena is modeled to be generalized Gaussian distributed with $\gamma=0.7$ [1]. For such distributions, the smaller the values of $\gamma$, the sharper the pdf's. This intuitively implies that the entropies with the same variance are 
TABLE I

FINDING $\delta_{H}^{C_{1}}$ WITH ENTROPY

BOUND $=1.5$ BY THE SECANT METHOD

\begin{tabular}{c||c|c|c|c|c|c}
\hline Lena & 1 & 2 & 3 & 4 & 5 & 6 \\
\hline$\delta_{a}$ & 0.5000 & 0.5000 & 0.5000 & 0.5000 & 0.5000 & 0.5000 \\
\hline$\delta_{b}$ & 4.0000 & 1.7971 & 1.1766 & 1.0605 & 1.0407 & 1.0377 \\
\hline$\delta_{n}$ & 1.7971 & 1.1766 & 1.0605 & 1.0407 & 1.0377 & \\
\hline Entropy & 1.1912 & 1.4589 & 1.5015 & 1.5083 & 1.5093 & \\
\hline Error & 4.8510 & 5.4308 & 5.5701 & 5.5954 & 5.5993 & \\
\hline \hline Mandrill & 1 & 2 & 9 & 10 & 11 & 12 \\
\hline$\delta_{a}$ & 1.0000 & 1.0000 & 1.0000 & 1.0000 & 2.8359 & 2.8359 \\
\hline$\delta_{b}$ & 6.0000 & 4.4141 & 2.8367 & 2.8363 & 2.8363 & 2.8360 \\
\hline$\delta_{n}$ & 4.4141 & 3.4463 & 2.8363 & 2.8359 & 2.8360 & \\
\hline Entropy & 1.3046 & 1.4450 & 1.5196 & 1.5196 & 1.5196 & \\
\hline Error & 15.7052 & 16.8517 & 17.9989 & 18.0003 & 18.0000 & \\
\hline
\end{tabular}

TABLE II

Finding $\delta_{H}^{C_{2}}$ WITH ENTROPy Bound $=1.5$ BY THE FALSE Position Method

\begin{tabular}{c||c|c|c|c|c|c}
\hline Lena & 1 & 3 & 5 & 7 & 8 & 9 \\
\hline$\delta_{a}$ & 10.000 & 9.3169 & 9.2983 & 9.2937 & 9.2933 & 9.2933 \\
\hline$\delta_{b}$ & 4.0000 & 9.0811 & 9.2919 & 9.2919 & 9.2919 & 9.2930 \\
\hline$\delta_{n}$ & 9.0811 & 9.2983 & 9.2950 & 9.2933 & 9.2930 & \\
\hline Entropy & 0.2094 & 0.2006 & 0.2007 & 0.2008 & 0.2009 & \\
\hline Error & 5.5134 & 5.6026 & 5.6017 & 5.6007 & 5.5997 & \\
\hline \hline Mandrill & 1 & 2 & 3 & 4 & 5 & 6 \\
\hline$\delta_{a}$ & 10.0000 & 10.0000 & 10.0000 & 10.0000 & 9.3708 & 9.3708 \\
\hline$\delta_{b}$ & 6.0000 & 9.0588 & 9.3330 & 9.3633 & 9.3633 & 9.3707 \\
\hline$\delta_{n}$ & 9.0588 & 9.3330 & 9.3633 & 9.3708 & 9.3707 & \\
\hline Entropy & 0.7399 & 0.7173 & 0.7151 & 0.7144 & 0.7145 & \\
\hline Error & 17.6591 & 17.9606 & 17.9901 & 18.0002 & 17.9994 & \\
\hline
\end{tabular}

TABLE III

Finding $\delta_{E}^{D_{1}}$ WITH ERROR BOUNDS $=5.6$ AND 18 IN LENA AND MANDRILL, RESPECTIVELY, BY THE FALSE POSITION METHOD

\begin{tabular}{c||c|c|c|c|c|c|c}
\hline Lena & 1 & 2 & 3 & 4 & 5 & 6 & 7 \\
\hline$\delta$ & 0.2000 & 0.2608 & 0.3229 & 0.3389 & 0.3413 & 0.3415 & 0.3415 \\
\hline Entropy & 1.3834 & 1.4411 & 1.4879 & 1.4984 & 1.4999 & 1.5000 & 1.5000 \\
\hline Error & 6.9569 & 6.8475 & 6.7353 & 6.7057 & 6.7014 & 6.7010 & 6.7011 \\
\hline \hline Mandrill & 1 & 2 & 3 & 4 & 5 & 6 & 7 \\
\hline$\delta$ & 0.2000 & 0.4899 & 0.7429 & 0.8701 & 0.9199 & 0.9267 & 0.9274 \\
\hline Entropy & 1.2041 & 1.3621 & 1.4539 & 1.4870 & 1.4984 & 1.4999 & 1.4999 \\
\hline Error & 27.1937 & 25.9317 & 24.8407 & 24.3260 & 24.1259 & 24.0999 & 24.0982 \\
\hline
\end{tabular}

reduced when the values of $\gamma$ decrease. When the values of $\gamma$ decrease from 1 to 0.7 , the reduction of the entropy variance follows from (13) and is shown in Fig. 6. Explicitly, the Shannon bound is lower. We also observe that the rate distortion function in Fig. 7 is closer to the Shannon bound than that in Fig. 10. This is reasonable since Laplacian distribution $(\gamma=1)$ is more similar to the Gaussian distribution $(\gamma=2)$ than the histogram
TABLE IV

FINDING $\delta_{E}^{D_{2}}$ WITH ERROR BOUNDS $=5.6$ AND 18 IN LENA AND MANDRILL, ReSPECTIVELY, By the FALSE POSITION METHOD

\begin{tabular}{c||c|c|c|c|c|c}
\hline Lena & 1 & 2 & 3 & 4 & 5 & \\
\hline$\delta_{a}$ & 0.5000 & 0.7405 & 1.0039 & 1.0039 & 1.0039 & \\
\hline$\delta_{b}$ & 4.0000 & 4.0000 & 4.0000 & 1.0698 & 1.0658 & \\
\hline$\delta_{n}$ & 0.7405 & 1.0039 & 1.0698 & 1.0658 & & \\
\hline Entropy & 1.5801 & 1.5205 & 1.4987 & 1.5000 & & \\
\hline Error & 6.0214 & 5.6435 & 5.5599 & 5.5645 & & \\
\hline \hline Mandrill & 1 & 2 & 9 & 10 & 11 & 12 \\
\hline$\delta_{a}$ & 1.0000 & 1.1603 & 3.0099 & 3.0100 & 3.0101 & 3.0101 \\
\hline$\delta_{b}$ & 8.0000 & 8.0000 & 8.0000 & 8.0000 & 8.0000 & 3.0102 \\
\hline$\delta_{n}$ & 1.1603 & 1.5802 & 3.0100 & 3.0101 & 3.0102 & \\
\hline Entropy & 1.5427 & 1.5802 & 1.5000 & 1.5000 & 1.5000 & \\
\hline Error & 23.1597 & 21.6122 & 17.6352 & 17.6352 & 17.6350 & \\
\hline
\end{tabular}

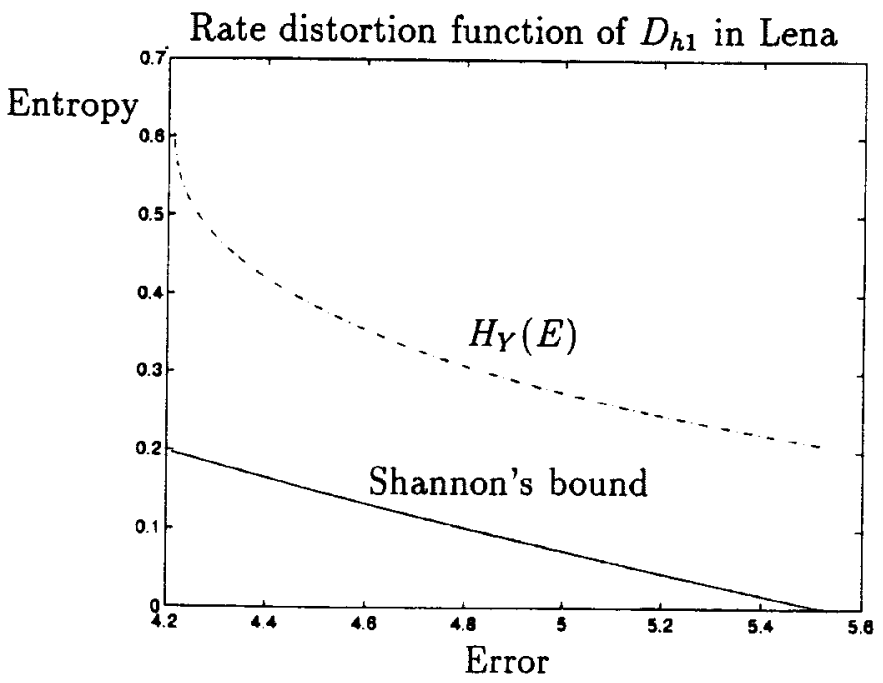

Fig. 10. Minimum entropy function with different error bounds is greater than the Shannon bound by about 0.2 bits/pixel in the case of $D_{h 1}$ of Lena.

of $D_{h 1}(\gamma=0.7)$. This reveals that a better performance in the sense of rate distortion functions will be attained for smaller values of $\gamma$. The results are consistent with those obtained from the energy concentration viewpoint. As a result, we are able to acquire a good compression ratio for smaller values of $\gamma$. This result also demonstrates the potential contribution of DWT and indicates that DWT is a popular and adaptive transform in the fields of data compression.

\section{CONCLUSION}

Through a three-level scalar quantizer performed after the DWT, a global maximum entropy can be observed. From this point, we are able to discuss minimum quantization error with entropy constrained and minimum entropy with an error bound, which are useful in real implementations. The numerical approaches of the secant and false position methods are provided to obtain the solutions to these problems from a geometrical point of view, and two testbed images (Lena and Mandrill) are implemented to verify the results. The rate distortion function of 
a three-level scalar quantizer is also addressed. Because of the inherency of the minimum quantization error in vector quantization, it is noteworthy to find the minimum entropy when the mean squared error of the quantization has been specified.

\section{APPENDIX}

\section{PROOF OF THEOREM 5.1}

From (13) and changing variable $(1 / \gamma)$ by $\beta$, we have

$$
V_{0}(\beta)=\frac{2 \sigma^{2} \Gamma^{3}(\beta) e^{2 \beta} \beta^{2}}{\pi e \Gamma(3 \beta)} .
$$

Sequentially, we define $V_{1}(\beta)=\left(\Gamma^{3}(\beta) e^{2 \beta} \beta^{2} / \Gamma(3 \beta)\right)$. Taking the derivative of $V_{1}(\beta)$ with respect to $\beta$, we get (14), shown at the bottom of the page. Since $\Gamma^{\prime}(\beta)=\left[-\eta+\sum_{n=1}^{\infty}((1 / n)-\right.$ $(1 / \beta+n-1))] \Gamma(\beta)$, where $\eta$ is the so-called Euler's constant and is equal to $\lim _{k \rightarrow \infty}(1+(1 / 2)+\cdots+(1 / k)-\ln k)=$ $0.57721 \ldots,(14)$ can be rewritten as

$$
\begin{aligned}
& \frac{d V_{1}(\beta)}{d \beta} \\
& =\frac{\beta \Gamma^{2}(\beta) e^{2 \beta}}{\Gamma^{2}(3 \beta)}\left\{\left[2 \Gamma(\beta)+3 \beta\left(-\eta+\sum_{n=1}^{\infty} \frac{1}{n}\right.\right.\right. \\
& \left.\left.\quad-\frac{1}{\beta+n-1}\right) \Gamma(\beta)+2 \beta \Gamma(\beta)\right] \Gamma(3 \beta) \\
& \left.\quad-3 \beta \Gamma(\beta)\left(-\eta+\sum_{n=1}^{\infty} \frac{1}{n}-\frac{1}{3 \beta+n-1}\right) \Gamma(3 \beta)\right\} \\
& =\frac{\beta \Gamma^{2}(\beta) e^{2 \beta}}{\Gamma^{2}(3 \beta)}\left[2 \beta+2-3 \beta \sum_{n=1}^{\infty} \frac{1}{\beta+n-1}-\frac{1}{3 \beta+n-1}\right] \\
& =\frac{\beta \Gamma^{2}(\beta) e^{2 \beta}}{\Gamma^{2}(3 \beta)}\left[2 \beta+2-3 \beta \sum_{m=0}^{\infty} \frac{1}{\beta+m}-\frac{1}{3 \beta+m}\right] \\
& \left.=\frac{\beta^{2} \Gamma^{2}(\beta) e^{2 \beta}}{\Gamma^{2}(3 \beta)}\left[2-3 \sum_{m=1}^{\infty} \frac{1}{\beta+m}-\frac{1}{3 \beta+m}\right] \cdot\right]
\end{aligned}
$$

For convenience, we define $V_{2}(\beta)=2-3 \sum_{m=1}^{\infty}(1 / \beta+m)-$ $(1 / 3 \beta+m)$, and the result of $V_{2}(1 / 2)=0$ is obtained directly.

Moreover, we are going to show that $V_{2}(\beta)$ is a strictly decreasing function of $\beta$. For $\beta_{1}>\beta_{2}$, then

$$
\begin{aligned}
& V_{2}\left(\beta_{1}\right)-V_{2}\left(\beta_{2}\right) \\
= & 3\left(\beta_{1}-\beta_{2}\right)\left[\sum_{m=1}^{\infty} \frac{1}{\left(\beta_{1}+m\right)\left(\beta_{2}+m\right)}\right. \\
& \left.-\sum_{m=1}^{\infty} \frac{3}{\left(3 \beta_{1}+m\right)\left(3 \beta_{2}+m\right)}\right]
\end{aligned}
$$

$$
\begin{aligned}
= & 3\left(\beta_{1}-\beta_{2}\right)\left[\sum_{m=1}^{\infty} \frac{3 \times 3}{\left(3 \beta_{1}+3 m\right)\left(3 \beta_{2}+3 m\right)}\right. \\
& -\sum_{l=1}^{\infty} \frac{3}{\left(3 \beta_{1}+3 l\right)\left(3 \beta_{2}+3 l\right)} \\
& -\sum_{l=1}^{\infty} \frac{3}{\left(3 \beta_{1}+3 l-1\right)\left(3 \beta_{2}+3 l-1\right)} \\
& \left.-\sum_{l=1}^{\infty} \frac{3}{\left(3 \beta_{1}+3 l-2\right)\left(3 \beta_{2}+3 l-2\right)}\right] \\
= & 3\left(\beta_{1}-\beta_{2}\right)\left\{\left[\sum_{m=1}^{\infty} \frac{3}{\left(3 \beta_{1}+3 m\right)\left(3 \beta_{2}+3 m\right)}\right.\right. \\
& \left.-\sum_{l=1}^{\infty} \frac{3}{\left(3 \beta_{1}+3 l-1\right)\left(3 \beta_{2}+3 l-1\right)}\right] \\
& \left.+\left[\sum_{m=1}^{\infty} \frac{3}{\left(3 \beta_{1}+3 m\right)\left(3 \beta_{2}+3 m\right)}\right]\right\} \\
& \left.\left.-\sum_{l=1}^{\infty} \frac{3}{\left(3 \beta_{1}+3 l-2\right)\left(3 \beta_{2}+3 l-2\right)}\right]\right\} \text { since the two terms in braces are negative. } \\
< & 0,
\end{aligned}
$$

Hence, $V_{2}(\beta)$ is a monotonically decreasing function. By the fact that $V_{2}(1 / 2)=0$, we notice that $V_{2}(\beta)$ is positive for $\beta<$ 0.5 and negative for $\beta>0.5$. As a result, we obtain that i) $V_{1}(\beta)$ is a strictly increasing function of $\beta$ for $\beta<0.5$, and ii) $V_{1}(\beta)$ is a strictly decreasing function of $\beta$ for $\beta>0.5$. In other words, the entropy variance $V_{0}(\gamma)$ possesses the property of monotonic increment when $\gamma<2$. Conversely, it has the property of monotonic decrement when $\gamma>2$. Consequently, the global maximum of the entropy variance occurs as the value of $\gamma$ reaches 2, and this is the case of Gaussian distribution.

\section{REFERENCES}

[1] M. Antonini, M. Barlaud, P. Mathieu, and I. Daubechies, "Image coding using wavelet transform," IEEE Trans. Image Processing, vol. 1, pp. 205-220, Apr. 1992

[2] A. N. Akansu and M. J. T. Smith, Subband and Wavelet Transforms. Boston, MA: Kluwer, 1996.

[3] P. J. Burt and E. H. Adelson, "The Laplacian pyramid as a compact image code," IEEE Trans. Commun., vol. COMM-36, pp. 532-540, 1983.

[4] T. Berger, "Optimum quantizers and permutation codes," IEEE Trans. Inform. Theory, vol. IT-18, pp. 759-765, 1972.

[5] P. A. Chou, T. Lookabaugh, and R. M. Gray, "Entropy-constrained vector quantization," IEEE Trans. Acoust., Speech, Signal Processing, vol. 37, pp. 31-42, Jan. 1989.

[6] I. Daubechies, "Orthonormal bases of compactly supported wavelets," Commun. Pure Appl. Math., vol. 41, pp. 906-966, 1988.

[7] I. Daubechies, Ten Lectures on Wavelets. Philadelphia, PA: SIAM, 1992.

[8] H. Gish and J. N. Pierce, "Asymptotically efficient quantizing," IEEE Trans. Inform. Theory, vol. IT-14, pp. 676-683, 1968.

$$
\begin{aligned}
\frac{d V_{1}(\beta)}{d \beta} & =\frac{\left[2 \beta \Gamma^{3}(\beta) e^{2 \beta}+\beta^{2} 3 \Gamma^{2}(\beta) \Gamma^{\prime}(\beta) e^{2 \beta}+\beta^{2} \Gamma^{3}(\beta) e^{2 \beta} 2\right] \Gamma(3 \beta)-\beta^{2} \Gamma^{3}(\beta) e^{2 \beta} \Gamma^{\prime}(3 \beta) 3}{\Gamma^{2}(3 \beta)} \\
& =\frac{\beta \Gamma^{2}(\beta) e^{2 \beta}\left\{\left[2 \Gamma(\beta)+3 \beta \Gamma^{\prime}(\beta)+2 \beta \Gamma(\beta)\right] \Gamma(3 \beta)-3 \beta \Gamma(\beta) \Gamma^{\prime}(3 \beta)\right\}}{\Gamma^{2}(3 \beta)} .
\end{aligned}
$$


[9] J. D. Hoffman, Numerical Method for Engineers and Scientists. New York: McGraw-Hill, 1992.

[10] J. S. Lim, Two-Dimensional Signal and Image Processing. Englewood Cliffs, NJ: Prentice-Hall, 1990

[11] D. G. Luenberger, Linear and Nonlinear Programming, 2nd ed. Reading, MA: Addison-Wesley, 1990.

[12] M. J. Maron, Numerical Analysis: A Practical Approach, 2nd ed. New York: Macmillan, 1987.

[13] S. G. Mallat, "A theory for multiresolution signal decomposition: The wavelet representation," IEEE Trans. Pattern Anal. Machine Intell., vol. 11, pp. 674-693, 1989.

[14] J. Max, "Quantizing for minimum distortion," IRE Trans. Inform. Theory, vol. IT-6, pp. 7-12, 1960.

[15] A. N. Netravali and R. Saigal, "Optimum quantizer design using a fixedpoint algorithm," Bell Syst. Tech. J., vol. 55, pp. 1423-1435, 1976.

[16] P. Noll and R. Zelinski, "Bounds on quantizer performance in the low bit-rate region," IEEE Trans. Commun., vol. COMM-26, pp. 300-304, 1978.

[17] A. Papoulis, Probability, Random Variables, and Stochastic Processes, 3rd ed, Singapore: McGraw-Hill, 1991

[18] A. Pentland and B. Horowitz, "A practical approach to fractal-based image compression," in Proc. Data Compres. Conf., 1991, pp. 176-185.

[19] G. M. Roe, "Quantizing for minimum distortion," IEEE Trans. Inform. Theory, vol. IT-10, pp. 384-385, 1964

[20] F. Scheid, Numerical Analysis, 2nd ed, Singapore: McGraw-Hill, 1989

[21] C. E. Shannon, "Coding theorems for a discrete source with a fidelity criterion," in IRE Nat. Conv. Rec., 1959, pp. 142-163.

[22] G. Strang and T. Nguyen, Wavelets and Filter Banks. Cambridge, MA: Wellesley-Cambridge, 1996.

[23] M. Vetterli and J. Kovačević, Wavelets and Subband Coding. Englewood Cliffs, NJ: Prentice-Hall, 1995.

[24] B.-F. Wu and H.-H. Hsu, "The global minimum of scalar quantization errors by discrete wavelet transforms in image compression," Proc. Nat. Sci. Coun., pt. A, vol. 23, no. 2, pp. 223-234, 1999.

[25] — - "Statistical properties and entropy reduction by discrete wavelet transforms," in Proc. Int. Symp. Multi-Technol. Inform. Process. Hsinchu, Taiwan, R.O.C., Dec. 1996, pp. 283-288.

[26] R. C. Wood, "On optimum quantization," IEEE Trans. Inform. Theory, vol. IT-15, pp. 248-252, 1969.

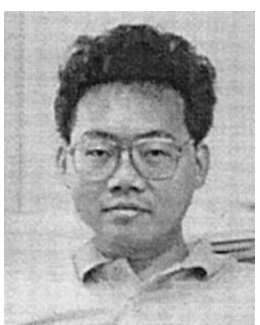

Bing-Fei Wu received the B.S. and M.S. degrees from the National Chiao Tung University, Hsinchu, Taiwan, R.O.C. in 1981 and 1983, respectively, and the Ph.D. degree in electrical engineering, majoring in control theory and signal processing, from the University of Southern California, Los Angeles, in 1992.

From 1983 to 1984, he was with the Institute of Control Engineering, National Chiao Tung University, as an Assistant Researcher, working on optimal control theory and digital control systems. From 1985 to 1988 , he was with the Department of Communication Engineering at the same university as a Lecturer. Since 1992, he has been with the Department of Electrical and Control Engineering, where he is currently a Professor. As an active industry consultant, he also involved with flash memory controller design, multimedia applications, and portable computer peripheral design. His research interests include chaotic systems, fractal signal analysis, image/video coding, wavelet analysis and applications, and control system design in optical mechanisms.

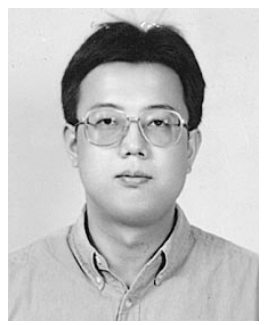

Hung-Hseng Hsu was born in KinMen, Fujian, Taiwan, R.O.C. in 1967. He received the B.S. and M.S. degrees in control engineering from the National Chiao Tung University, Hsinchu, Taiwan, in 1989 and 1990, respectively, and the Ph.D. degree in electronic engineering from the National Chiao Tung University in 1997.

$\mathrm{He}$ is currently an engineer with the Computer and Communications Research Laboratories, Industrial Technology Research Institute. His research interests include image coding, video coding, video phone systems, with associated communication protocols, VoIP, and cable modem system implementation. 